\title{
Brain Computer Interface
}

\author{
Boulevard of Smarter Thoughts
}

\author{
Sumit Ghulyani , Yashasvi Pratap, Sumit Bisht, Ravideep Singh \\ Department Of Computer Science \& Engineering \\ Dronacharya College of Engineering \\ Gurgaon, Haryana, India
}

\begin{abstract}
The Brain Computer Interface is a major breakthrough for the technical industry, medical world, military and the society on a whole. It is concerned with the control of devices around us such as computing gears \& even automobiles in the near future without really the physical intervention of the user. It helps bridge the communication gap between the society and the disabled. This mainly lays its focus on people suffering from brainstem stroke, going through a spinal cord injury or even blindness. BCI helps such patients to retain or restore communication with the outside world through intelligent signals from the brain due to the high risk of paralysis under such circumstances. This is achieved by a signal acquisition technique and converting these signals available from the sensors placed on the scalp into real-time computer commands that can be visually operated and understood. It has nothing to do with the natural neural transmission of brain signals but extracts them with the help of sensors to be processed and direct the outputs to an external device.

This may also prove to be a major military gadget where troops may communicate their thoughts in highly stressed situations without breaking the hush. But, as every technology have some merits and demerits, so does BCI.
\end{abstract}

Keywords- Brain Computer Interface (BCI); Blood Oxygen Level Dependent (BOLD); Electrocorticography (ECoG); Practical Electrical Stimulation (PES); Electroencephalograph (EEG); Magnetic Resonance Imaging (MRI),Functional Magnetic Resonance Imaging (fMRI) brain scythe, motor cortex.

\section{OVERVIEW}

A plethora of thoughts is ready to open up and make things work just fine as they did ever. In the human brain, the thoughts and emotions are transmitted in the form of electric signals with the help of those millions of neurons. The following figure below displays a wireless neuro-headset developed by Emotiv Systems which detects brainwave signals and categorizes them specifically into conscious \&nonconscious thoughts

This makes possible selected processing of only appropriate signals that are meant to be transmitted by the user [1]. This device makes use of the same set of electrodes as sensors which are used in the medical examination known as electroencephalograph, or in short EEG. The display is maintained by an advanced software program than the old EEG paper \& pen tracker scheme.

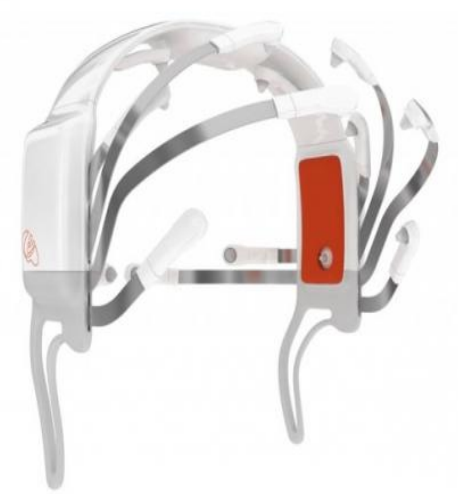

Figure 1. BCI Developed by Emotiv Systems

MRI or Magnetic Resonance Imaging technique is under research to be used in BCIs as an effective approach to map actual part of the brain with the task to be performed. With Brain Computer Interface, the control of the world, on a whole, lays in the hands of thoughts \& emotions. The power behind the advent of this technology is the working of the human brain itself. Though a person maybe disabled with speech, hearing or a total "locked-in", the brain never stops functioning. This feature is made use of in BCI using external peripheral gears to detect these signals and convert them into visual commands that can be read and understood. In the course of this, the actions of the brain are manipulated to control the computing gears and communication devices instead of generating signals using the traits of a motor movement.

The figure 2 is a depiction of the BrainGate technology which is a part of the research carried on at the Brown Institute for Brain Science. It aims at implanting a baby sensor in the gray matter which will read the electric signals and transmit them to the computer system. Evenly, the research in the field of Human Computer Interaction has achieved impressive results in the rapid \& heavy transmission of signals from the computer to the human brain using sensory channels and vice versa. A phase of perceptual computing is about to begin which studies user's psychology and operating environment to process the information about a user's intent. 


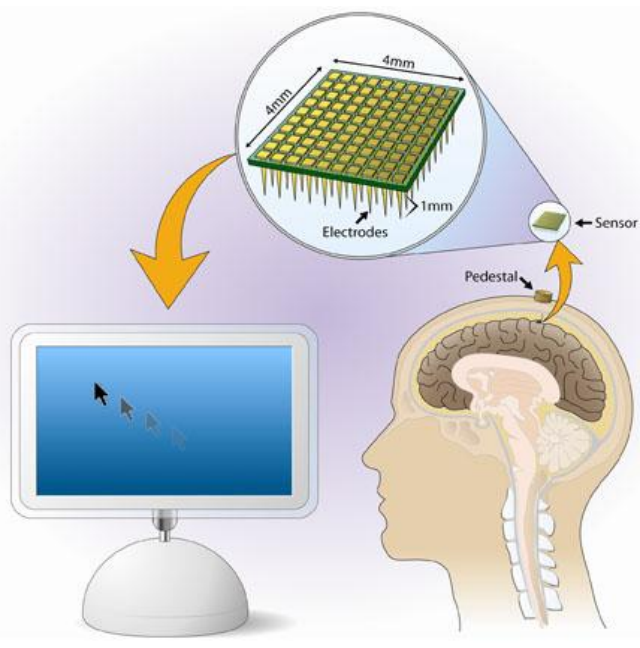

Figure 2. Chip Implantation

In this way, a computer can adapt itself according to the user and the task to be performed thereby giving phenomenal results. If extensive research is done and one succeeds in recognizing the signals transmitted when light falls on the retina it may lead to a major breakthrough of $\mathrm{BCI}$ in the medical world which may grant artificial vision to the blind. And as we see, possibilities are endless.

\section{HISTORY}

The past of the Brain Computer Interface can be dated back to the time when Electroencephalography was in his early years of birth. This became possible with the successful research of German scientist Hans Berger, who in 1924, succeeded in recording the electrical signals in the human brain. He successfully analyzed the oscillatory activity of the brain and discovered the Alpha wave, also popularly known as the Berger's wave. Though his instrumentation, which consisted of Lippmann capillary electrometer and silver wires, was very desolate and led to unsuccessful results in advanced phases, he was able to mark the start of a revolution. Success was later achieved with the help of Siemens double coil recording galvanometer which made recording of electric signals as small as one ten thousandth of a volt. Berger's preliminary work led to success in elimination of various diseases related to brain cells and the advancement of the Electroencephalography or EEG, in short. The actual birth of the first relative to Brain Computer Interface took place in 1969 at the University Of Washington, School of Medicine (UWSOM), Seattle [2]. A monkey was used as the subject where he successfully deflected a biofeedback meter measuring arm with the intervention of his neural activity. Another subsequent research used the same subject, but in numbers to announce the voluntary control of solo and multiple neurons in the primary cortex. Many research groups have been able to record the neurological stimuli and depict them with the various body movements thus controlled.

A major breakthrough was achieved when Philip Kennedy were successful in implanting electrodes in a monkey's cortex. With the passage of time, a change in the subject was brought when monkeys and rats were replaced by cats for further research issues at Berkeley when visual images were reproduced by decoding the neural shots in the cat's brain. In this process, the electrodes were implanted in the region of the dual lobed grey matter called the thalamus, which records the actions perceived by the retina. The generation and reconstruction of the data was hack done by gradually synthesizing and decoding the signals obtained over a period of time with the visual perceptions involved.

The human intervention as subjects took place in Japan when researchers implanted electrodes to a patient's scalp and similar results were achieved. A further research at the University of Pittsburgh led to the use of even fewer neurons than ever in a working Brain Computer Interface. They brought about a jaw-breaker advancement which displayed a monkey's brain activity to control a robotic arm that fed him with fruits kept beside him. Research groups all around the globe are working towards applying kinematics to the concept of BCIs to retain normal muscle movement of the patient by stimulating the muscles electrically.
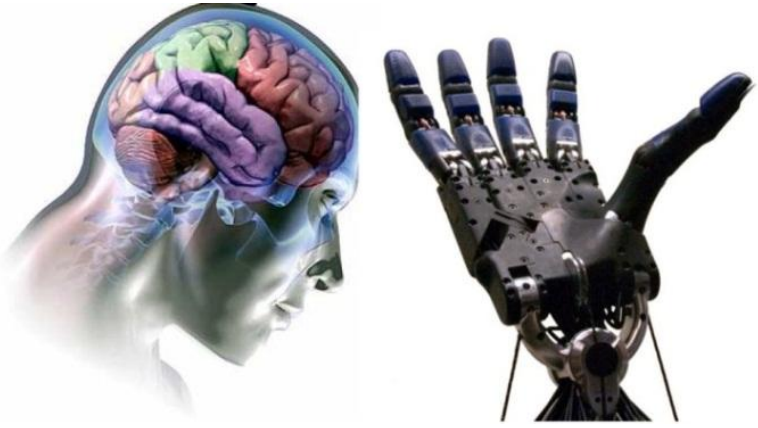

Figure 3. Association of brain signals with movement of hand

\section{OPERATIONAL TRAITS}

The performance of a BCI is completely based on the approach our intellect functions. Our brain is crammed with neurons, entity nerve cells allied to one another by dendrites and axons that bear electronic signals all through the entire body. Every time we think, budge, sense or consider something, our neurons are at labour. That work is carried out by these miniature electric signals that whiz from neuron to neuron as prompt as $250 \mathrm{mph}$. The signals are generated by differences in electric prospective carried by ions on the membrane flanked by two neurons. Although the routes the signals take are insulated by something called myelin, some of the electric signal escape [3].

Scientists with the exploit of modern technology can perceive those signals, construe what they mean and utilize them to direct a device of some breed. It can also work the other way around. For example, researchers could outline out what signals are sent to the brain by the diverse sensory organs for example, the signals in the aural nerve can be traced and the task of hearing can be mapped. An even more remarkable use of this technology is that the signals can be generated to be sent to the brain and a enthused sense of hearing can be induced in the subject even if one is deaf. One of the biggest challenges in front of brain-computer interface researchers at the moment is the basic technicalities of the interface itself. The sleekest and least detrimental is a set of electrodes, an apparatus known as an electroencephalograph (EEG) which is attached to the scalp. 

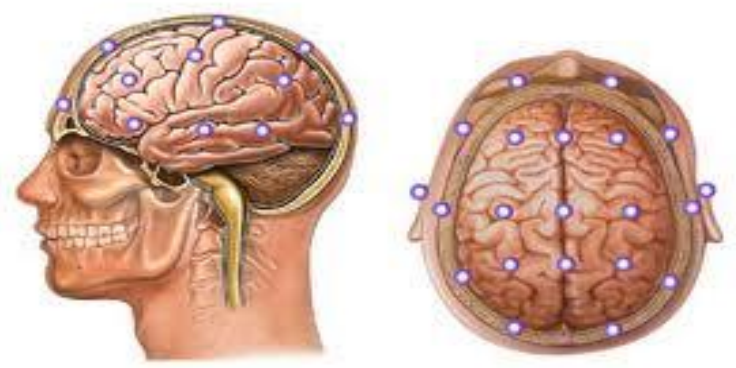

Figure 4. Electroencephalogram (EEG) scan

The electrodes interpret brain signals. However, the cranium restricts a lot of electrical signal, and distorts whatever does get through it.

As a resolution, scientists may implant electrodes directly into the brain, or on the facade of the brain. This aids in higher reception of electric signals and allows electrode positioning in the particular area where those explicit signals are generated. The electrodes assess minuscule differences in the voltage amid neurons. The signals are then augmented and interpreted by a computer program. In the case of an infix $\mathrm{BCI}$, the method is overturned. A mainframe will renovate a signal, like from a tape, into the voltages that would elicit neurons. The signal generated from a peripheral apparatus is sent to an electroencephalograph (EEG) detector installed in the relevant quarter of the brain. The neurons in the head are enthused with these signals and the subject obtains an illustration, reverberation or a related sensory familiarity parallel to whatsoever gear is used.

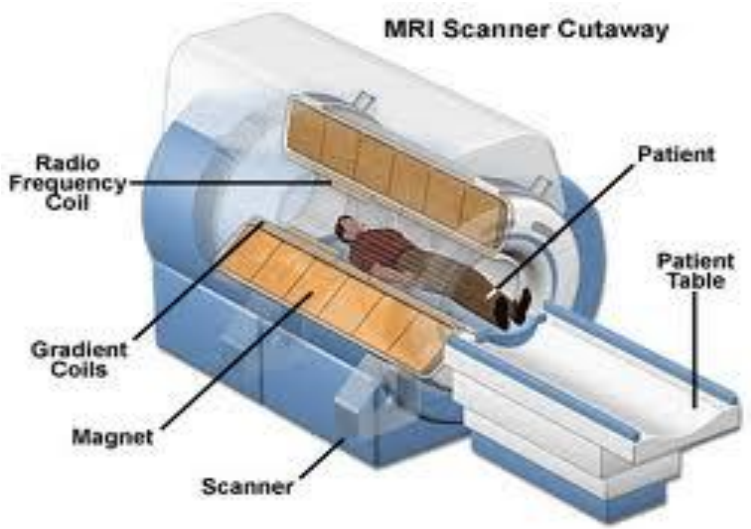

Figure 5. MRI Scanner

A substitute approach to scrutinize and assess brain bustle is using a Magnetic Resonance Image (MRI). The MRI setup is a colossal and convoluted apparatus. It produces high decree imagery of the brain, but it can't be used as component of an enduring BCI. Researchers use it to swot up definite brain functions or to plot in the brain the quarter where electrodes should be sited to evaluate an unambiguous task.

\section{FMRI}

Functional Magnetic Resonance Imaging or more popularly fMRI is a non-invasive, much similar technique as the MRI or Magnetic Resonance Imaging, the only difference being the basic measure of BOLD i.e. Blood Oxygen Level
Dependent in fMRI. It is advancement to MRI and measures brain activity, but by detecting changes in the flow of blood.

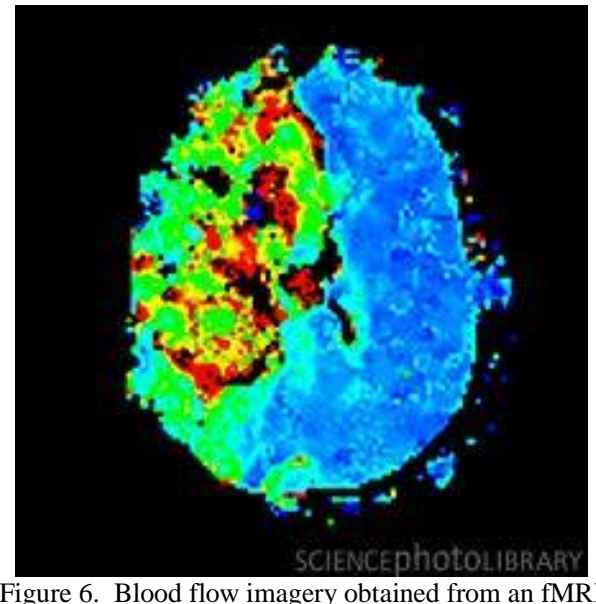

With this advancement, on might just sneak in someone else's brain and derive its thoughts and even detect lies. The basic idea behind this technology originated from the fact that blood rich in oxygen behaves differently to a magnetic field than deoxygenated samples. In other words, both have different magnetic resonance traits. The more active parts of a brain receive higher flow of oxygenated blood. The procedure for the test is similar to the present MRI and uses only magnetic fields and radio waves. Though no radiations are used, it is highly advised not to be performed during pregnancy, and on people with artificial limbs or pacemaker [4].

This technique is medically used rarely but to monitor the growth of tumors and regulate the normal functioning of the brain after a stroke. Brain Mapping is an emerging field for fMRI which will make Brain Computer Interfaces even superior as it determines the areas of brain that control the various body movements and moreover on planning surgery and market survey \& research.

Functional Magnetic Resonance Imaging (fMRI) also possesses certain vantages and detriments. The major breaker is that this technology is still in its birth phase and the researchers know very less regarding the overall working of the apparatus. Moreover, the setup is really expensive to be used for research and brain computer interfaces. To continue, it works only when the subject lays still and thus a lot of work needs to be done.

The major breakthrough that gets it going is the absence of any radiation in any phase of the scan as in X-ray scan and PET and the positive feedback and response from the tests carried out. Though scores of tests resulted in a higher level of bewilderment amongst the researchers since fMRI relates to the blood flow only and not on the individual neuron functioning in the brain and the blood flow measure was not enough to critically examine and complement on the results thus obtained.

\section{BCI SPEECH}

The BCI system can be trained what an individual's brain scrutiny or EEG looks like when one is focused on a fussy 
article, since the electrical oomph in the brain changes in proportion to what the individual is doing. The rise of Electrocorticography (ECoG) has been a new hint podium for braincomputer interface systems. Consequently, it was anonymous whether further neuro-physiological substrates, such as the vocalization net, might be used to further develop on or harmonize on motor-based power paradigms. For the foremost instance, that ECoG signals allied with diverse, blatant and anticipated phoneme enunciation can facilitate invasively monitored patients to manage a one-dimensional computer pointer precisely.

This stuff was discernible within upper gamma regularity oscillations and enabled clients to realize concluding object accuracies flanked by $68 \%$ and $91 \%$ in 15 minutes [5]. In addition, one of the patients achieved full-bodied control via recordings from a micro array that consisted of $1 \mathrm{~mm}$ spaced micro wires. These results proposed that the cortical system allied with verbal communication could present an added cognitive substrate for BCI maneuver. Subsequently, these signals can be recorded from a cortical assortment that is minute and austerely invasive.

\section{APPLICATIONS}

\section{A. Brain scythe}

It's trouble-free to scythe a computer, but nearly everyone always considered that hacking the human intellect was unfeasible. As seen in science fiction movies and novels like the Matrix, Brainstorm \& Surrogates, play around with gaining admittance to the human mind. But, researchers have finally confirmed the leeway to hack the human brain [6]. The researchers used a brain mainframe interface, usually used in following a line of investigation that scans brain patterns.

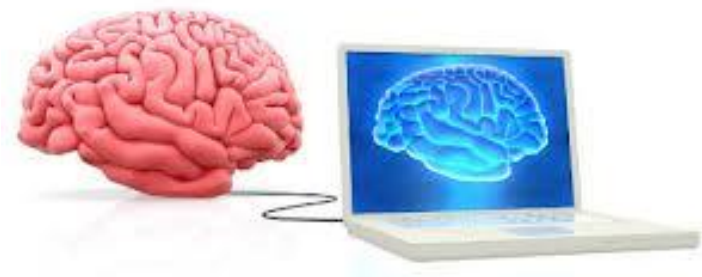

Figure 7. Brain Scythe

The contemporary models consent to users to be in charge of their computers with their thoughts, but the researchers have verified that it goes well both ways. The panel built a part of custom software that can, in essence, interpret the mind. They were proficient enough to effectively use the software pooled with the brain scanner to mine susceptible data including credit card PINs, address, DOB and other.

\section{B. Restores spinal coordination}

Researchers at Chicago have lucratively bypassed the spinal flex and restored well motor control to paralyzed limbs by means of a brain-computer interface. The researchers have shaped a neuro-prosthesis that groups a BCI that is wired directly into 100 neurons in the motor cortex of the subject, and a Practical Electrical Stimulation (PES) tool that is wired into the brute muscle of the subject's upper limb. When the subject tries to shift his arm, the huddle of 100 neurons activates, creating a torrent of data which can be examined and analyzed by the BCI to foresee what muscles the subject is trying to budge, and with what intensity. This interpreted statistics is conceded to the PES, which then triggers the correct muscles to execute the preferred movement. The last part outcome is a mainframe network that in actual fact replaces the nervous structure and restores fine motor control to a paralyzed arm.

\section{Bionic prosthetic ogle}

A bionic prosthetic ogle is the mock eye for the sightless or a being with a hitch in seeing matter due to a defected retina. It speaks the tongue of the brain by transmitting the signals fired by the retina when something is seen. This is the sense in the wake of the prosthetic eye. Electric pulses are measured by attaching electrodes to an animal's optic nerves.

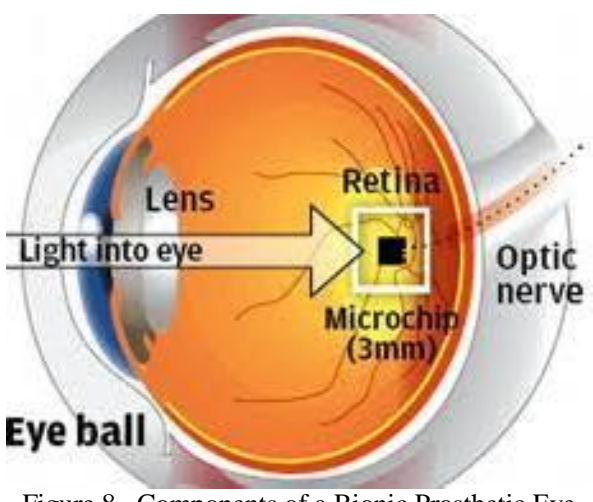

Figure 8. Components of a Bionic Prosthetic Eye

One might not know, but if a retina at all times generates the same electric cipher when looking at a building, and a dissimilar code when a being is in sight, work can be retreated to derive the retina's tangible programming procedure. This revolution would leave mankind startled when a silicon chip would be installed into the eye and then wired to the optic nerves.

\section{Restored neurological implementation}

Brain computer interface can facilitate the cases where flexibility has been smacked or got narrow by a stroke. Scientists in St. Louis have revealed the detection of the brain signals simply thinking on affecting a partly or wholly paralyzed limb. The damaged half of the brain that is responsible normally for moving the hand can no longer do so, so the hint arrives from the unharmed other half of the brain. To lay bare the prospective to help restore motion, scientists allied electrode-detected brain signals to the movement of a marker on the computer panel. Researchers are now working out a motorized glove which will turn all the imagination into a reality.

\section{DETRIMENTS}

Every technology suffers severe drawbacks, but it does not halt the research process, although slows down the speed at which advancements appear. The major breaker of this technology is that BCIs are still in crude form and need to be refined for the 'future of thoughts' to come alive. The 
development process is further slowed by certain ethical issues on the road to success. The complexity of the brain adds up to the massacre being equipped with a staggering 100 billion neurons, and detecting the association of the type of neurons with specific body movements looks arduous.

The structure and placement of electrodes needs a research in itself since the one placed outside the skull are unable to detect appropriate signal voltage to produce optimal results. Moreover, the electrodes implanted inside the skull cause damage to the tissues and may even lead to serious scars resulting in undesired effects

\section{VANTAGES}

But, as BCIs appear to be the gate to an era of thoughtful communication, the research goes on keeping in mind the extra edge its merits showcase despite its failures. Paralysis will never stay a curse as in the history of medical sciences since the patients will be able to communicate with their mates and docs through a graphical BCI. To add to it, they may even help in the movement of prosthetic limbs to sink the effect of any motion related impairment. Visual \& hearing impairments may become history as these interfaces act as artificial eyes \& ears transmitting data to and fro the human mind successively.

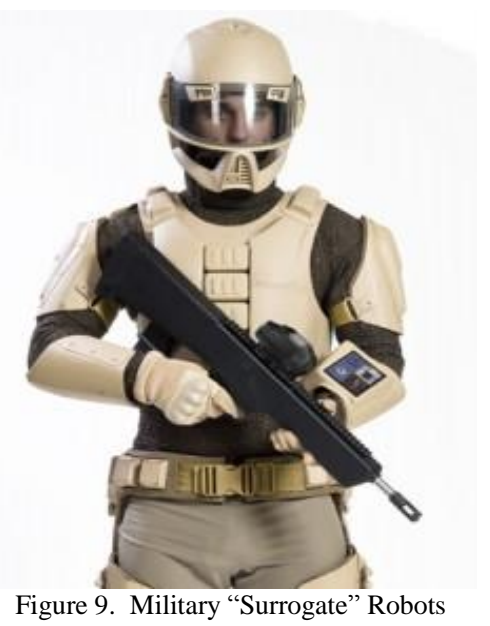

With gradual improvements, the armed forces may get even better armed with this interface. Communication can occur without any vocal disturbance, and who knows armed surrogates may come in effect very soon [7].

This will lead to less loss of men but money on the battlefield, as they are stationed far away and connected to their subject surrogate machines which operate in the main field and the troops live even if their surrogate is blown away by a grenade. This means a commandment of 1000 soldiers may fight all their life with different bodies. The world of entertainment is about to get vowed with this new technology, as gamers will now control their milieu with the might of their mind.

\section{PROPOSED WORK}

(i) Advancements in fMRI must occur so as to scan and process even the moving parts of subject and not the part which lies still.

(ii) Development of robotic limbs to provide co-ordination with the brain computer interface.

(iii) The electrodes to be used in the interface carcass should be made highly sensitive so as to provide exterior signal detection without the installation of the electrodes in the grey matter.

(iv) Combined tests to be conducted based on both MRI \& fMRI to determine collectively the association of different hotspots in the brain to diverse body movements by deriving the relationship between blood flow and neuron activity.

(v) Development of high resolution interface displays to record brain activity.

(vi) Provisions and techniques to restrict unauthorized enforced access to someone else's brain.

\section{CONCLUSION}

The research goes on and the possibilities of refinement are as vast as the universe, never ending. The maximum a human brain can think of what it can do is the result in the making of the American science scripture Surrogates (2009) directed by Jonathan Mostow [8].

The scripture shows how the world of humans turns into an era of surrogates which are none other than robotic bodies which are controlled by human brains from home. The surrogates are set to work almost everything physically, be it routine tasks or strategic war situations without any human intervention but mind control.

The world changes and divides into 2 parts - humans \& machines until the surrogate inventor himself devises a deadly weapon on seeing the far-sighted fate of his invention that starts wiping out the surrogates along with its users. But, this is as we know long way ahead in the future.

As of the birth of the Brain Computer Interface, it is bliss on medical grounds for the patients suffering from spinal cord injuries or impaired hearing or vision. A new era of thought has raised its head in this chaotic world which will help change the way of communication, since humans appeared first on earth.

Partial immobility or paralysis will disappear from the medical books as a curse. This can also be a major breakthrough for the armed forces to communicate with each other in highly stressed situations like war \& terrorism, but this may even lead to a major demerit if gone in wrong hands. By wrong hands I mean wrong minds which may use this boon as a bane against the human society as a whole. 
With progress in research, one may forget his very own mouse and keyboard, the somewhat most essential input components of a computer system as they are replaced with these interfaces. When applied to robotics, these interfaces may help physically challenged people to gain the same place in the society as any other normal person.

With advancements, BCIs may help such people to control robotic bodies with their brain to cope with their routine tasks. So, let the brain do the talking and send your jaw muscles on a vacation, just saying. Despite of all the detriments, the merits give the BCIs that extra edge which makes it a possible suitor for a better future.

\section{REFERENCES}

[1] Emotiv: EEG Systems http://emotiv.com/

[2] About BCIs http://inventors.about.com/od/bstartinventions/a/JohnDonoghue.htm

[3] HowStuffWorks: Brain Computer Interfaces http://computer.howstuffworks.com/brain-computer- interface.htm

[4] How fMRI Works http://science.howstuffworks.com/fmri.htm

[5] Using Electrocorticography speech for BCI http://www.ncbi.nlm.nih.gov/pubmed/21471638

[6] Gaining Access To Brain at $\$ 300$ only http://www.webpronews.com/your-brain-can-now-be-hacked-for-under200-2012-08

[7] Mind Controlled Robots http://www.extremetech.com/extreme/132389real-life-avatar-the-first-mind-controlled-robot-surrogate

[8] Surrogates (2009) http://www.imdb.com/title/tt0986263/plotsummary

\section{AUTHORS PROFILE}

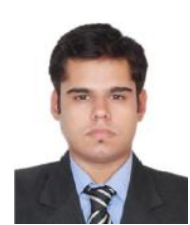

Sumit Ghulyani is presently a final year student pursuing degree in Bachelor of Technology in the field of Computer Science Engineering from Dronacharya College of Engineering, Gurgaon, India. Other than being the campus ambassador for a couple of organisations, he has been one of the soul limbs in successfully coordinating \& hosting technical events with Microsoft and HRs of various other technical giants. Authored a couple of research papers, the fields of his research interest include Brain Computer Interface, Windows Operating Systems, Apple MAC OS, Android Operating Systems, Wireless Power Transmission Artificial Intelligence \& Virtual Reality. Email: sumit.ghulyani@live.com, GSM: +91-9910550618

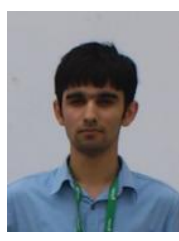

Yashasvi Pratap is a final year student pursuing degree in Bachelor of Technology in the field of Computer Science Engineering from Dronacharya College of Engineering, Gurgaon, India. Being a fresh seed in the field of research, his major interests include Computer Mediated Reality and Ubiquitous Computing. He is actively engaged in other activities which include interest in world geography, historical timeline \& zoology. Email: yashasvi000pratap@gmail.com, GSM: +919968052498

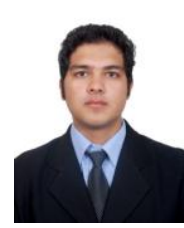

Sumit Bisht is a final year student pursuing degree in Bachelor of Technology in the field of Computer Science Engineering from Dronacharya College of Engineering, Gurgaon, India. A soul coordinator for several technical \& cultural events at college, he never halts at thriving for the best. Stepping forward to publish his second work of brilliance, his major interests include Programming Paradigms, ATM Networks \& Wireless Computing. Email: sumitbisht@live.com, GSM: +919968803069

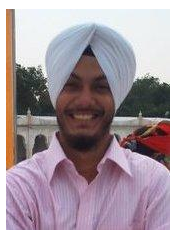

Ravideep Singh is a final year student pursuing degree in Bachelor of Technology in the field of Computer Science Engineering from Dronacharya College of Engineering, Gurgaon, India. Other than his proficiency in learning foreign languages, his major interests include Distributed Operating Systems, Artificial Intelligence \& Neural Networks. Email: ravideep singh@live.com,GSM: +919891912029 